\title{
Strain relaxation via formation of cracks in compositionally modulated two-dimensional semiconductor alloys
}

Hossein Taghinejad' ${ }^{1}$, Ali A. Eftekhar ${ }^{1}$, Philip M. Campbell ${ }^{2}$, Brian Beatty ${ }^{2}$, Mohammad Taghinejad ${ }^{1}$, Yao Zhou ${ }^{3}$, Christopher J. Perini' ${ }^{2}$, Hesam Moradinejad ${ }^{1}$, Walter E. Henderson ${ }^{4}$, Eric V. Woods ${ }^{4}$, Xiang Zhang ${ }^{5}$, Pulickel Ajayan ${ }^{5}$, Evan J. Reed ${ }^{3}$, Eric M. Vogel ${ }^{2}$ and Ali Adibi $^{1}$

Composition modulation of two-dimensional transition-metal dichalcogenides (TMDs) has introduced an enticing prospect for the synthesis of Van der Waals alloys and lateral heterostructures with tunable optoelectronic properties. Phenomenologically, the optoelectronic properties of alloys are entangled to a strain that is intrinsic to synthesis processes. Here, we report an unprecedented biaxial strain that stems from the composition modulation of monolayer TMD alloys (e.g., $\mathrm{MoS}_{2 x} \mathrm{Se}_{2(1-x)}$ ) and inflicts fracture on the crystals. We find that the starting crystal $\left(\mathrm{MoSe}_{2}\right)$ fails to adjust its lattice constant as the atoms of the host crystal (selenium) are replaced by foreign atoms (sulfur) during the alloying process. Thus, the resulting alloy forms a stretched lattice and experiences a large biaxial tensile strain. Our experiments show that the biaxial strain relaxes via formation of cracks in interior crystal domains or through less constraint bounds at the edge of the monolayer alloys. Griffith's criterion suggests that defects combined with a sulfur-rich environment have the potential to significantly reduce the critical strain at which cracking occurs. Our calculations demonstrate a substantial reduction in fracture-inducing critical strain from $11 \%$ (in standard TMD crystals) to a range below $4 \%$ in as-synthesized alloys.

npj 2D Materials and Applications (2018)2:10 ; doi:10.1038/s41699-018-0056-4

\section{INTRODUCTION}

Transition-metal dichalcogenides (TMDs) are the most extensively explored family of two-dimensional (2D) materials beyond graphene. The non-vanishing bandgap of the TMDs enables a wide range of optoelectronic devices including photo-detectors, ${ }^{1,2}$ light-emitting diodes, ${ }^{3,4}$ and lasers. ${ }^{5}$ A diverse range of available transition metals (e.g., $\mathrm{M}=\mathrm{Mo}$ and $\mathrm{W}$ ) and chalcogens (e.g., $\mathrm{X}=\mathrm{S}$ and $\mathrm{Se}$ ) offers a "digital" portfolio of 2D binary crystals in the form of $\mathrm{MX}_{2}$ with functionalities over a broad spectral range from visible to near infrared. To make this portfolio a "continuum", the alloying of binary crystals through composition modulation on either metal $\left(M^{\prime}{ }_{x} M_{1-x} X_{2}\right)$ or chalcogen $\left(M^{\prime}{ }_{2 x} X_{2(1-x)}\right)$ sites has been pursued. In fact, modulating the composition ratio $(0<x<1)$ continuously tunes the bandgap energy of the ternary alloy between the characteristic bandgaps of the two constituting binary compounds. For instance, several recent studies have demonstrated fine-tuning the bandgap energy in $\mathrm{Mo}_{x} \mathrm{~W}_{1-x} \mathrm{Se}_{2}$ between $1.5 \mathrm{eV}\left(\mathrm{MoSe}_{2}\right)$ and $1.7 \mathrm{eV}\left(\mathrm{WSe}_{2}\right)^{6,7}$ in $\mathrm{WS}_{2 x} \mathrm{Se}_{2(1-x)}$ between $1.7 \mathrm{eV}\left(\mathrm{WSe}_{2}\right)$ and $2.1 \mathrm{eV}\left(\mathrm{WS}_{2}\right)^{8}$ in $\mathrm{MoS}_{2 x} \mathrm{Se}_{2(1-x)}$ between $1.5 \mathrm{eV}\left(\mathrm{MoSe}_{2}\right)$ and $1.9 \mathrm{eV}\left(\mathrm{MoS}_{2}\right)_{1,10}$ and in $\mathrm{Mo}_{x} \mathrm{~W}_{1-x} \mathrm{~S}_{2}$ between $1.9 \mathrm{eV}$ (MoS2) and $2.1 \mathrm{eV}\left(\mathrm{WS}_{2}\right) .^{11,12}$ Moreover, the spatial modulation of the chemical composition allows for the formation of complex lateral heterostructures with sharp interfaces and tunable inter-junction band offsets. ${ }^{10,13-16}$ The realization of lateral heterostructures is particularly important as it enables rectifying current-voltage (I-V) characteristics, ${ }^{13,16,17}$ which is currently hardly achievable in 2D TMDs because of the lack of wellstablished doping mechanisms in atmospheric conditions.
The ternary alloys of 2D TMDs can be synthesized using two general approaches: (i) a direct chemical vapor deposition (CVD)based technique in which the simultaneous reaction of three precursors yields ternary alloys ${ }^{7-10}$ and (ii) an indirect two-step technique in which a $\mathrm{MX}_{2}$ binary compound forms in the first step and later serves as a host that accommodates the atoms of the third element $\left(\mathrm{M}^{\prime}\right.$ or $\left.\mathrm{X}^{\prime}\right)$ in the second step (alloying step). ${ }^{16,18,19}$ Although the direct approach yields high quality 2D alloys, it is not an optimal method for realizing lateral heterostructures as it provides minimal spatial control over the chemical composition. In contrast, the indirect two-step approach can be combined with standard lithography processes to deterministically form lateral heterostructures with intended dimensions in predefined locations. ${ }^{16}$ Moreover, the two-step alloying allows the post-growth trimming of optoelectronic properties in 2D TMD crystals. In the two-step technique, however, adding atoms (e.g., $\mathrm{X}^{\prime}$ ) reside in a relatively stable lattice of a pre-grown binary crystal $\left(M_{2}\right)$ that does not necessarily hold an optimal lattice constant for an intended alloy with non-zero composition ratio ' $x$ ' (as in $M X^{\prime}{ }_{2 x} \mathrm{X}_{2(1}$ x). Consequently, the two-step nature of this approach might stimulate a biaxial strain in the synthesized ternary alloy, which influences optoelectronic properties in an unpredictable manner. Thus, understanding the effect of the two-step composition modulation on the optical and structural properties of TMD alloys is an integral part of designing novel devices based on the alloys and lateral heterostructures of 2D TMD crystals. As current studies have largely overlooked this important subject, a comprehensive study is in high demand.

\footnotetext{
${ }^{1}$ School of Electrical and Computer Engineering, Georgia Institute of Technology, Atlanta, GA 30332, USA; ${ }^{2}$ School of Materials Science and Engineering, Georgia Institute of Technology, Atlanta, GA 30332, USA; ${ }^{3}$ Department of Materials Science and Engineering, Stanford University, Stanford, CA 94305, USA; ${ }^{4}$ Institute of Electronics and Nanotechnology (IEN), Georgia Institute of Technology, Atlanta, GA 30332, USA and ${ }^{5}$ Department of Materials Science and Nanoengineering, Rice University, Huston, TX 77005, USA

Correspondence: Ali Adibi (ali.adibi@ece.gatech.edu)
}

Received: 17 January 2018 Revised: 23 March 2018 Accepted: 2 April 2018

Published online: 30 April 2018 

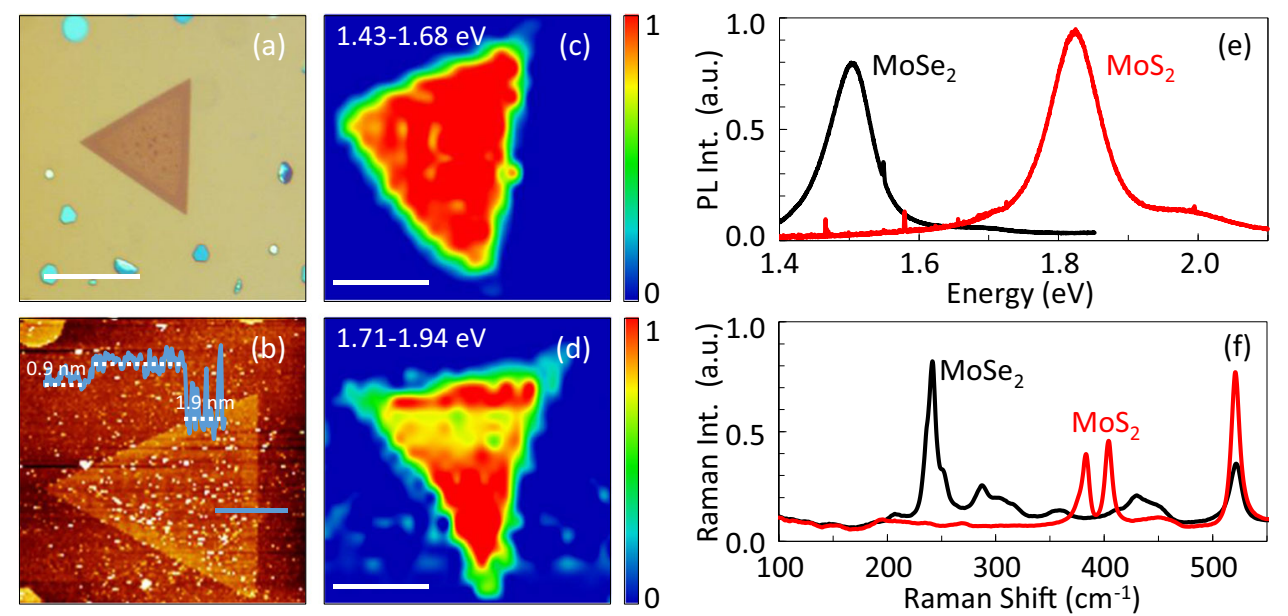

Fig. 1 Conversion of $\mathrm{MoSe}_{2}$ to $\mathrm{MoS}_{2}$. a Optical and $\mathbf{b}$ AFM images of a representative $\mathrm{MoSe}_{2}$ crystal before the sulfurization step. The inset of

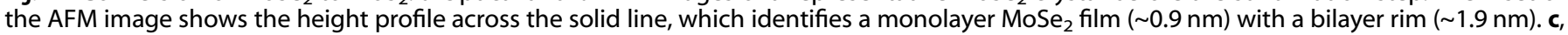
d The PL maps of the starting $\mathrm{MoSe}_{2}$ and converted $\mathrm{MoS}_{2}$ crystals, respectively. Color-bars represent normalized PL intensity integrated over the energy ranges that are marked in the maps. The PL map of the converted $\mathrm{MoS}_{2}$ crystal in the emission energy range of $1.43-1.68 \mathrm{eV}$ is shown in Figure S2 of SI. e, f Representative PL and Raman spectra of the pristine $\mathrm{MoSe}_{2}$ and converted MoS ${ }_{2}$, respectively. Scale bars in a, c, and $\mathbf{d}$ are 20,10 , and $10 \mu \mathrm{m}$, respectively

Here, using a monolayer $\operatorname{MoS}_{2 x} \mathrm{Se}_{2(1-x)}$ material platform, we show that the indirect nature of the two-step composition-modulation technique inherently yields a synthesis of strained TMD alloys with disintegrated crystal structures. Our two-step alloying approach employs a standard CVD method for the synthesis of monolayer $\mathrm{MoSe}_{2}$ crystals (first step) followed by a high-temperature sulfurization step (i.e., second step) that incorporates $\mathrm{S}$ atoms into the host lattice of $\mathrm{MoSe}_{2}$. A structural analysis demonstrates that the resulting compositionally modulated alloys are mostly cracked and that the shape and propagation path of the cracks depend on the composition ratio (i.e., $x$ ). Using systematic PL spectroscopy and mapping, the origin of the cracks is identified to be a biaxial tensile strain that stems from the replacement of Se atoms by $S$ atoms during the sulfurization step. Our calculations, based on the Griffith's method, shed light on the critical role of pre-existing cracks and crystal defects in weakening the fracture strength of the as-synthesized Van der Waals alloys. To magnify the effect of the biaxial strain on compositionally modulated crystals, throughout this paper, we will focus on the full conversion of the $\mathrm{MoSe}_{2}$ films to $\mathrm{MoS}_{2}$ (i.e., $x=1$ ), unless mentioned otherwise. We believe that the unprecedented results of the observations made in this study can be extended to other 2D semiconductor alloys synthesized via two-step composition-modulation techniques.

\section{RESULTS AND DISCUSSION}

Our two-step composition-modulation approach employs a previously reported CVD technique for the growth of $\mathrm{MoSe}_{2}$ films in the first step. ${ }^{20}$ Then, in the second step, we proceed with the sulfurization of $\mathrm{MoSe}_{2}$ crystals using a high-temperature annealing process under a sulfur vapor ambient that replaces selenium (Se) atoms with sulfur (S) atoms. To prevent cross-contamination effects, each step is performed in a dedicated furnace exclusively exposed to either Se or S vapors. For a detailed understanding and optimization of the process, we change sulfurization parameters (e.g., temperature and time) and then characterize samples using $\mathrm{PL}$ and Raman spectroscopy (the experimental details and the optimization results are provided in Supporting Information (SI)). We found that after sulfurization at $\sim 1000^{\circ} \mathrm{C}$ for $10 \mathrm{~min}$, the pristine $\mathrm{MoSe}_{2}$ monolayer crystals (Fig. 1a, b) are fully converted to $\mathrm{MoS}_{2}$, as verified via PL mappings before (Fig. 1c) and after (Fig. 1d) conversion. The representative PL spectrum of the converted
$\mathrm{MoS}_{2}$ (Fig. 1e) reveals that the emission energies of A-excitons and B-excitons at 1.82 and $1.97 \mathrm{eV}$, respectively, match those of CVDgrown and exfoliated $\mathrm{MoS}_{2}$ films reported in the literature. ${ }^{21,22}$ Thus, the utilized conversion process yields monolayer $\mathrm{MoS}_{2}$ crystals with optical qualities comparable to those of a pristine CVD-grown $\mathrm{MoS}_{2}$ crystal. The spatial mapping of the PL peak position (not shown here) verifies the uniform conversion of $\mathrm{MoSe}_{2}$ into $\mathrm{MoS}_{2}$, with only $50 \mathrm{meV}$ variation in the emission energy of the A-exciton across a converted sample. Using Raman spectroscopy, we draw a similar conclusion (Fig. 1f) and observe that the characteristic peaks of the pristine $\mathrm{MoSe}_{2}$ crystal at $241 \mathrm{~cm}^{-1} \quad\left(A_{1 \mathrm{~g}}\right.$ mode) and $288 \mathrm{~cm}^{-1}\left(E^{1}{ }_{2 \mathrm{~g}}\right.$ mode) completely vanished; instead, the characteristic peaks of $\mathrm{MoS}_{2}$ emerge at $382 \mathrm{~cm}^{-1}\left(\mathrm{E}_{2 \mathrm{~g}}^{1}\right.$ mode) and $405 \mathrm{~cm}^{-1}\left(\mathrm{~A}_{1 \mathrm{~g}}\right.$ mode). Therefore, the $\mathrm{PL}$ and Raman characterizations decisively determine that during the sulfurization step, the employed conversion process successfully supports the complete replacement of Se atoms by $\mathrm{S}$ atoms, which agrees with thermodynamical predictions. ${ }^{23}$

To show the potential of the indirect alloying technique for the synthesis of $\mathrm{MoSe}_{2}-\mathrm{MoS}_{2}$ lateral heterostructures, we protect parts of the starting $\mathrm{MoSe}_{2}$ film with a mask and selectively convert the unprotected regions to $\mathrm{MoS}_{2}$. The protecting mask can be designed using a standard lithography step followed by the deposition of the protection material (see the Methods Section for details). Figure $2 a$ represents an example of lateral heterostructures in which we have used an array of 70-nm-thick $\mathrm{SiO}_{2}$ lines to locally protect the starting $\mathrm{MoSe}_{2}$ crystals during the sulfurization process. To investigate the formation of the lateral heterostructures after the conversion process, we carry out line-scanned Raman mapping on the patterned sample (Fig. 2b). The alternating presence of the characteristic Raman peaks of $\mathrm{MoS}_{2}$ and those of $\mathrm{MoSe}_{2}$, in exposed and protected regions, respectively, verifies the formation of $\mathrm{MoSe}_{2}-\mathrm{MoS}_{2}$ heterostructures within the plane of the monolayer crystal. We also note that the $70 \mathrm{~nm}$-thick $\mathrm{SiO}_{2}$ mask is quite effective for protecting the $\mathrm{MoSe}_{2}$ crystal as the Raman spectra of the starting $\mathrm{MoSe}_{2}$ films remain intact in protected regions.

To gain more insight into the structural properties of the converted $\mathrm{MoS}_{2}$ crystals, we inspect the samples via scanning electron microscopy (SEM). In Fig. 3, we simultaneously identify three types of films across the samples: (i) entirely continuous (Fig. $3 a-c)$, (ii) entirely cracked (Fig. 3d-f), and (iii) partially cracked (Fig. $3 g-i)$ films. The SEM inspection of multiple independently 

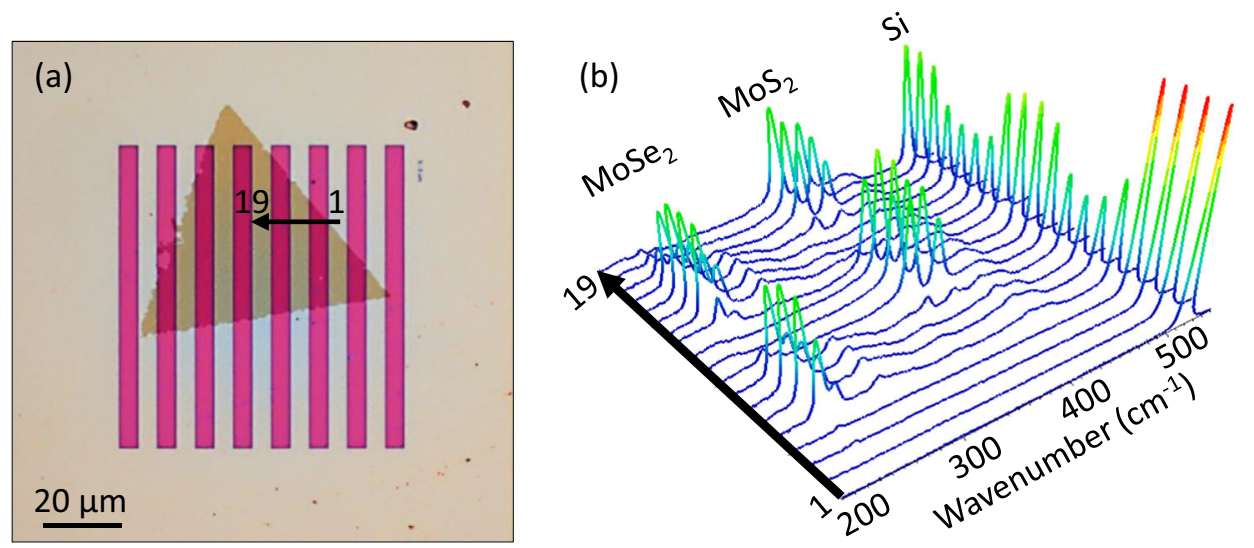

Fig. 2 Formation of $\mathrm{MoSe}_{2}-\mathrm{MoS}_{2}$ lateral heterostructure using patterning and selective sulfurization. a The optical image of the fabricated structure, where $\mathrm{SiO}_{2}$ lines (70-nm-thick, 5- $\mu \mathrm{m}$-wide, and 10- $\mu \mathrm{m}$-pitch) are used to locally protect pristine $\mathrm{MoSe}_{2}$ triangle during the sulfurization process. The $\mathrm{SiO}_{2}$ lines appear magenta in the optical image. $\mathbf{b}$ The Raman line mapping along the arrow that is shown in a. The characteristic Raman peaks of $\mathrm{MoSe}_{2}\left(241\right.$ and $\left.288 \mathrm{~cm}^{-1}\right)$ and those of $\mathrm{MoS}_{2}$ (382 and $405 \mathrm{~cm}^{-1}$ ) appear alternatively along the scanned line, which verifies the formation of the $\mathrm{MoSe}_{2}-\mathrm{MoS}_{2}$ lateral heterostructure. The Raman peak of silicon at $520 \mathrm{~cm}^{-1}$ is used as a refence in all spectra

converted samples consistently confirms that more than $\sim 90 \%$ of the $\mathrm{MoS}_{2}$ monolayer crystals are entirely cracked (including the one used in Fig. 1) and the rest are mostly continuous, with only a few partially cracked flakes on each sample. It is worth noting that prior to the conversion step, the pristine $\mathrm{MoSe}_{2}$ crystals are all continuous and cracks form only after the sulfurization process. We believe that the observed cracks stem from an induced strain that might originate from two sources: (i) the substitution of Se atoms by $\mathrm{S}$ atoms, and/or (ii) the thermal-expansion coefficient mismatch between monolayer films and the $\mathrm{SiO}_{2}$ substrate. To distinguish between these two sources, we repeat our standard conversion process with no sulfur load so that monolayer crystals experience similar thermal treatment while no composition conversion occurs. In this experiment, to account for the state of the monolayer crystal at the beginning $\left(\mathrm{MoSe}_{2}\right)$ and the end $\left(\mathrm{MoS}_{2}\right)$ of the conversion process, we simultaneously anneal CVDgrown monolayer $\mathrm{MoSe}_{2}$ and $\mathrm{MoS}_{2}$ samples. The SEM inspections (Figure S3, SI) do not display the formation of cracks in any of the as-treated samples. However, analogous to previous reports, ${ }^{24,25}$ we observe that high temperature annealing generates rather straight corrosion lines at the boundaries of crystal domains, marked by arrows in Fig. 3. Such corrosion lines should not be mistaken for cracks that form within individual crystal domains and span over significantly shorter ranges. Therefore, we conclude that the mismatch of thermal-expansion coefficients does not play a primary role, and the composition-modulation event governs the formation of cracks. In Figure S4 of the SI, we show that similar cracks form in the unprotected regions of 2D monolayer heterostructures (i.e., between $\mathrm{SiO}_{2}$ strips), where composition modulation occurs.

An examination of cracked crystals reveals an important trend that cracks consistently branch out of Y-shaped (triple) junctions or from the edge of monolayer crystals (e.g., see Fig. 3d). This observation suggests the existence of specific crack nucleation sites and preferential crystallographic directions over which cracks spread within the plane of monolayer crystals. Given that the origin of the strain is composition conversion, we think that unsaturated bonds (at the edges) or defect sites (within the interior domains of the film) serve as local sites at which the incorporation of $\mathrm{S}$ atoms into the lattice of $\mathrm{MoSe}_{2}$ is initiated. A similar contribution of defect sites in the formation of cracks and Y-shaped branching behavior has been recorded in TMD $^{26}$ films subjected to biaxial strain.

The propagation path of cracks between Y-shaped junctions carries valuable information regarding conversion-induced strain.
In the determination of the crack propagation path, the spatial distribution of the strain field at the tip of a propagating crack is a key factor. In addition, the Van der Waals interaction of monolayer crystals with the substrate provides a medium for the strain fields of individual cracks to mutually interact, defining a net propagation path between the Y-shaped junctions. Theoretical modeling based on molecular dynamics ${ }^{27}$ and classical fracture mechanics ${ }^{26}$ are effective for the qualitative prediction of the crack propagation path in monolayer $\mathrm{MoS}_{2}$ crystals under biaxial strain. In these models, the strain field is assumed to extend to a critical radius of $\left(r_{\mathrm{c}}\right)$ from the tip of the propagating cracks. As schematically shown in Figure S5 (in $\mathrm{SI}$ ), cracks propagate between two individual Y-shaped junctions via a curved or straight path if $r_{\mathrm{c}}$ is a large or small value, respectively. To change the $r_{\mathrm{c}}$ value, we change the relative strength of the strain by means of varying the composition ratio, $x$, in $\mathrm{MoSe}_{2 x} \mathrm{~S}_{2(1-x)}$ alloys in which increasing $x$ monotonically increases the biaxial strain. Thus, we prepare two sets of samples: (i) a fully converted crystal (Fig. 3j) with $x=1$ (i.e., large strain and large $r_{c}$ ) and (ii) a partially converted crystal (Fig. 3k) with $x \approx 0.5$ (i.e., small strain and small $r_{\mathrm{c}}$ ). A comparison of the SEM images of these samples shows that our experimental observations match the theoretical modeling; that is, in the fully converted sample, cracks mostly propagate via curved paths while in the partially converted sample, cracks obtain straight propagation paths (Fig. 3k). Furthermore, in the partially converted sample, cracks mostly form hexagonal domains and propagate along zigzag directions (the inset of Fig. 3k). This observation is also in agreement with molecular dynamics predictions, which identify the zig-zag direction as the most feasible propagation path with a minimum surface energy. ${ }^{27}$ Therefore, the structural analysis solidifies our assumption about the primary role of the conversion process (i.e., substitution of Se atoms by S atoms) in the creation of the biaxial strain and the formation of cracks in compositionally-modulated monolayer alloys.

To quantitatively study the conversion-induced strain in converted $\mathrm{MoS}_{2}$ crystals, we carry out PL spectroscopy, in which probing the optical bandgap provides direct information about the type (tensile/compressive) and the strength of the strain. ${ }^{28-31}$ For this purpose, we use a partially cracked $\mathrm{MoS}_{2}$ crystal that enables a direct comparison between the emission spectra of cracked and continuous regions (Fig. $4 a$, b) within a single film. Mapping the emission energy at the maximum PL intensity (Fig. 4c) indicates that compared to cracked regions, the continuous 

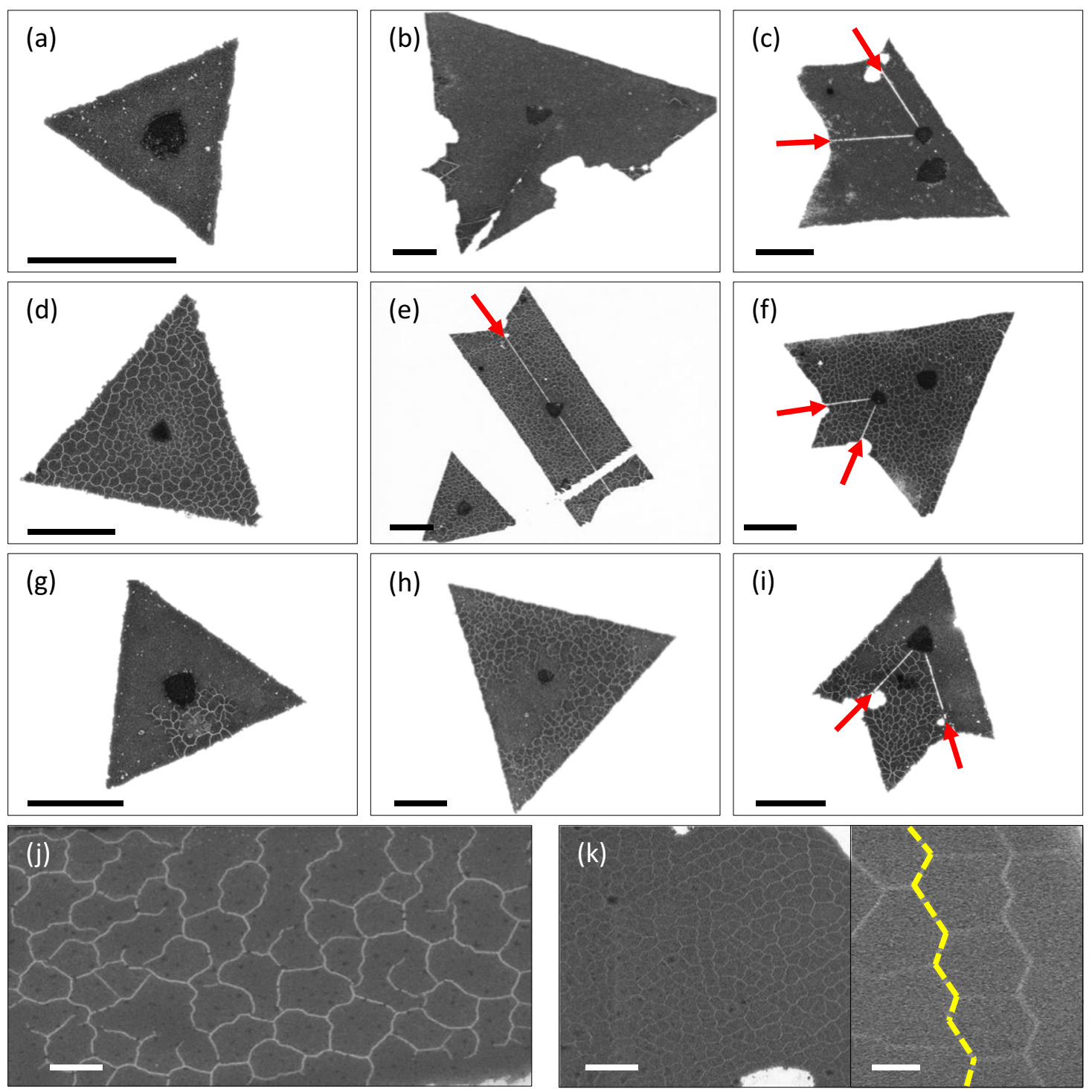

Fig. 3 Crack formation in converted $\mathrm{MoS}_{2}$ monolayer crystals. SEM inspection identifies three different types of morphologies among the converted $\mathrm{MoS}_{2}$ flakes; a-c entirely continuous, d-f entirely cracked, and $\mathbf{g}$-i partially cracked flakes. All the SEM images are collected from one sample after full conversion of the $\mathrm{MoSe}_{2}$ monolayer crystals to $\mathrm{MoS}_{2}$. Arrows point to the corrosion lines that form along the boundaries of the crystal domains. The effect of the conversion degree (i.e., ' $x$ ' in $\operatorname{MoS}_{2 x} \mathrm{Se}_{2(1-x)}$ ) is elaborated via comparing the crack propagation path in $\mathbf{j}$ a fully converted MoSe $\mathrm{e}_{2}$ crystal (i.e., $x=1$ ) and in $\mathbf{k}$ a partially converted film (with $x=0.5$ ). The inset of panel (k) highlights the zigzag path as the most energetically favorable direction for crack propagation in partially converted sample. Scale bars in a-i are $3 \mu \mathrm{m}$. Scale bars in $\mathbf{j}$, $\mathbf{k}$, and the inset of pane $(\mathbf{k})$ are $0.5,2$, and $0.3 \mu \mathrm{m}$, respectively

(i.e., crack free) regions emit light at lower energies. We also observe a significant drop in the intensity of the light emission in continuous regions (Fig. 4d). These two distinctions can be concurrently observed in the representative PL spectra of cracked and continuous films as displayed in Fig. 4e. Since the PL spectra of cracked regions imitate the standard emission spectrum of a pristine CVD-grown $\mathrm{MoS}_{2}$ film, we denote the continuous regions as the strained parts. Thus, the reduction of the optical bandgap and the drop in the emission intensity are taken as indications of a biaxial tensile strain that acts on continuous regions. ${ }^{31,32}$

Several groups have investigated the effect of biaxial tensile strain on the $\mathrm{MoS}_{2}$ bandgap, and a reduction of $\sim 100 \mathrm{meV}$ per $1 \%$ of strain is the most commonly reported value. ${ }^{31,32}$ Therefore, the bandgap reduction of $120 \pm 2 \mathrm{meV}$ (extracted from Fig. 4c) reflects a $1.2 \pm 0.2 \%$ biaxial tensile strain that acts on continuous regions. Since converted $\mathrm{MoS}_{2}$ regions remain continuous under this estimated strain value, cracking is expected to occur at biaxial tensile strains between $1.2 \pm 0.2 \%$ and a maximum expected value of $\varepsilon=\left(a_{\mathrm{MoSe}_{2}}-a_{\mathrm{MoSe}_{2}}\right) / a_{\mathrm{MoSe}_{2}} \approx 3.9 \% .{ }^{33,34}$ However, observation of cracks in the estimated range of alloying-induced biaxial strain (up to $\sim 3.9 \%$ ) is somewhat unexpected as previous studies show that TMD films tolerate up to $\sim 11 \%$ tensile strain before the crystal failure occurs. ${ }^{35}$ A simplistic insight into this discrepancy is provided by the well-known Griffith criterion, which predicts a reduction in fracture strength in the presence of defects or small cracks. $^{36,37}$ Based on the Griffith criterion, in the presence of a $2 /$ long pre-existing crack, the critical strain $\left(\varepsilon^{c}\right)$ can be estimated as $\varepsilon^{c}=\sqrt{2 \gamma / \pi E l}$, where $\gamma$ and $E$ are the surface energy density and the Young's modulus of the TMD crystal, respectively. As explained in detail in SI, we estimate a range of $E \approx 177-270 \mathrm{GPa}$ and an average value of $\gamma \approx 0.928 \mathrm{Joul} / \mathrm{m}^{2}$ (assuming no plasticity around the crack tip). Therefore, assuming two representative values of $I \approx 1 \mathrm{~nm}$ and $2 \mathrm{~nm}$, the calculated critical strains are $\varepsilon^{c} \approx$ $4.7-5.8 \%$ and $\varepsilon^{c} \approx 3.3-4 \%$, respectively, which fall within the 

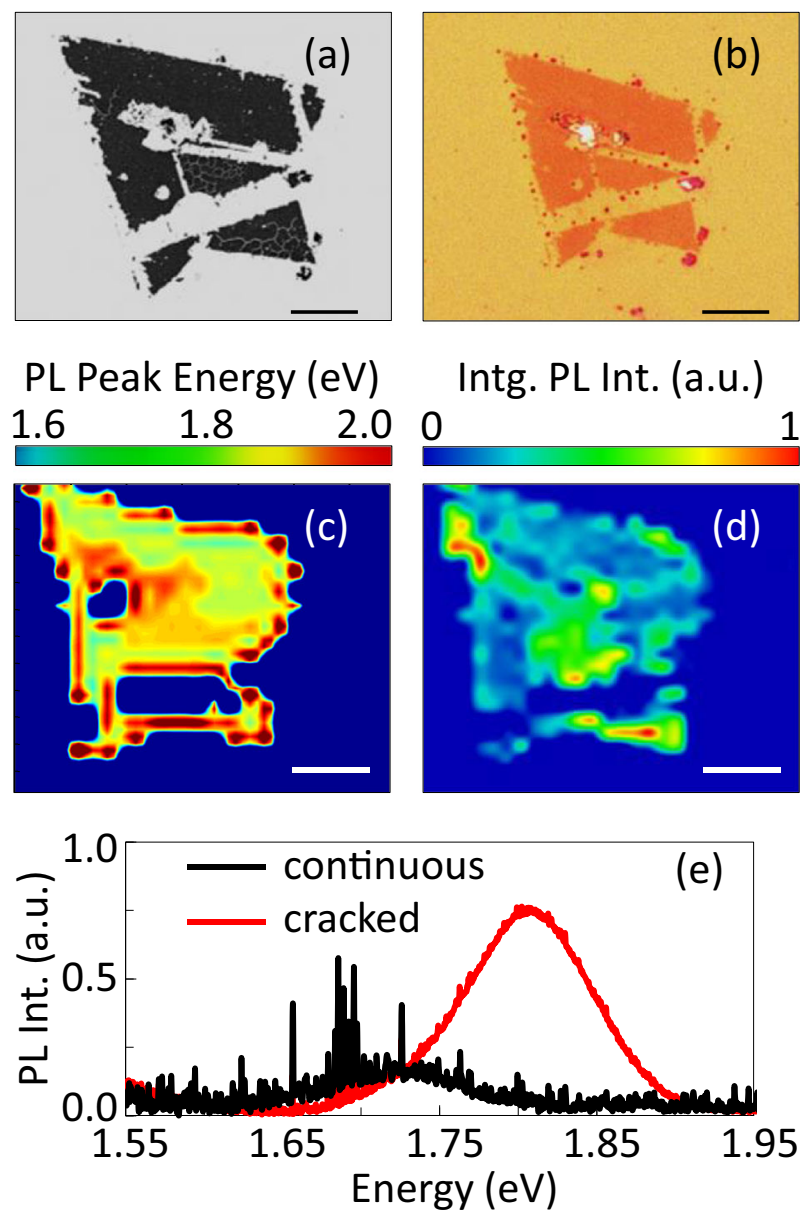

Fig. 4 Relaxation of the biaxial tensile strain via the formation of cracks in converted $\mathrm{MoS}_{2}$ crystals. a SEM and $\mathbf{b}$ optical images of a converted $\mathrm{MoS}_{2}$ monolayer crystal that is partially cracked. c Spatial mapping showing the PL energy at maximum PL intensity. The continuous regions demonstrate light emission at lower energies as compared to the emission from the cracked regions. The color bar depicts the energy span. d Spatial mapping of the PL intensity in which the continuous regions display low emission intensity. The normalized color bar represents the PL intensity integrated over an energy range of $1.6-2.0 \mathrm{eV}$ (corresponding to a wavelength range of 660-775 nm). e Representative PL spectra obtained from the cracked and continuous regions. Scale bars in a-d are $5 \mu \mathrm{m}$

expected range of alloying-induced biaxial strain. Based on insights provided by the Griffith criterion, the three different cracking morphologies (shown in Fig. 3) can be attributed to the heterogeneous distribution of the size/density of defects within starting $\mathrm{MoSe}_{2}$ crystals. In other words, only regions with a large defect size/density experience cracking, and defect-free regions remain continuous after the sulfurization step.

It is worth noting that reported first principle-calculations performed on graphene suggest that the Griffith criterion overestimates the critical strain of cracks shorter than $10 \mathrm{~nm} .^{37}$ Thus, smaller alloying-induced strain (e.g., in partially converted alloys with $x<1$ ) can also inflict the cracking of Van der Walls alloys. Indeed, defects severely reduce the critical strain of TMD crystals and cause the runaway propagation of cracks in a substantially smaller strain regime. Our conclusion closely agrees with a recently reported in-situ transmission electron microscopy study on monolayer $\mathrm{MoS}_{2}$, ${ }^{26}$ where defects were found to serve as initial sites from which cracks propagate along Y-shaped junctions, similar to our observations in Fig. 3. It is worth noting that our assumption regarding the length of the pre-existing cracks is consistent with recent experimental results that identify preexisting cracks with sizes ranging from a few nanometers up to several tens of nanometers in CVD-grown TMD atomic crystals. ${ }^{36}$ We also note that possible oxidation at the edges of cracks does not significantly contribute into differences observed in the PL spectra collected from cracked and continuous regions. In fact, our observed shift in the PL peak position of $\mathrm{MoS}_{2}$ films upon cracking is up to $140 \mathrm{meV}$ (Fig. 4), which is beyond the capacity of oxidation (i.e., $\sim 20 \mathrm{meV}^{38}$ ) for changing the emission spectrum of cracked $\mathrm{MoS}_{2}$ regions. A similar conclusion can be drawn using Raman measurements (see Figure S6, SI).

The assignment of tensile strain can be immediately verified as we note that the $\mathrm{MoS}_{2}$ crystal is synthesized via incorporating $S$ atoms into the $\mathrm{MoSe}_{2}$ film with a larger lattice constant $\left(a_{\mathrm{MoSe}_{2}}=\right.$ $3.288^{\circ} \mathrm{A}$ and $a_{\mathrm{MoS}_{2}}=3.160^{\circ} \mathrm{A}^{33,34}$ ). Indeed, the stable lattice of the starting crystal (i.e., $\mathrm{MoSe}_{2}$ ) fails to fully adjust its lattice constant as $\mathrm{Se}$ atoms are being replaced by the $\mathrm{S}$ atoms during the conversion step. Therefore, we speculate that the intermediate phases of the sulfurization step may produce $\operatorname{MoS}_{2 x} \mathrm{Se}_{2(1-x)}$ alloys (with $x \neq 0$ ) arranged in a crystal with a larger lattice constant, leading to a biaxial tensile strain that acts on the dynamically evolving alloy. Eventually, at some point, the built-up strain is released through the formation of cracks within the plane of the crystal. Considering the Se-Mo-Se vertically stacked configuration of the starting $\mathrm{MoSe}_{2}$ monolayer, we speculate that because of the Van der Waals interaction with the substrate, the incorporation of $\mathrm{S}$ atoms into the bottom Se-plane is more difficult than that into the top Se-plane. Such a substrate effect might contribute to the failure of the starting crystal when it adapts to an expected lattice constant. We also note that the edge sites of the converted $\mathrm{MoS}_{2}$ crystals display characteristics of a strain-free emission identical to those of the cracked regions (see Fig. $4 a, b$ ). This effect can be explained based on the access to the less constrained bonds at the vicinity of the $\mathrm{MoS}_{2}$ edge, which provides a pathway for relaxing the strain without the formation of cracks.

The optical properties of 2D TMDs are influenced by variations in the chemical composition of crystals, ${ }^{39}$ which raises the question about the role of possible composition heterogeneities in different PL spectra obtained from cracked and continuous regions. Thus, to map out the elemental composition of the cracked and continuous regions, we carry out time-of-flight secondary-ion mass spectroscopy (ToF-SIMS) with an SEM-level visualization capability and a sub-micron lateral resolution. For this study, we identify a region of the converted sample that contains continuous and cracked films (Fig. 5a) and then characterize it using the PL spectroscopy (Fig. 5b) prior to ToF-SIMS experiments. As illustrated in Fig. 5b, continuous regions emit light at lower energies and display a lower PL intensity, which is consistent with our measurements in Fig. 4. In ToF-SIMS experiments, we probe (i) Mo, (ii) S, and (iii) Se ions in positive (Fig. 5c) and negative (Fig. 5d) scans (positive/negative refers to the polarity of the ions). Elemental maps in Fig. 5c-i, d-ii demonstrate the homogeneous incorporation of Mo and $\mathrm{S}$ atoms in both cracked and continuous regions of the converted crystals, respectively. This observation rules out non-homogeneous composition conversion as a significant contributor to different PL spectra collected from cracked and continuous crystals. Moreover, the absence of Se atoms in positive (Fig. 5c-iii) and negative (Fig. 5d-iii) scans reflects the complete conversion of the $\mathrm{MoSe}_{2}$ crystals to $\mathrm{MoS}_{2}$ (within the limits of the experimental measurements). It is worth noting that ToF-SIMS spectroscopy provides a qualitative analysis of the elemental composition rather than a stoichiometric-level analysis (see Figure S8, SI). This is due to the difficulties of preparing standard calibration samples to serve as benchmarks for estimating the precise stoichiometry of the sample under test. However, our qualitative analysis in Fig. 5 remains reliable as far as the "relative" presence of the elements in cracked and continuous regions is concerned. However, the similarity of the emission 


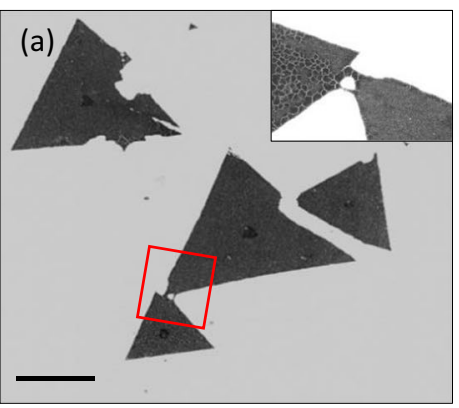

(c)

(i) $\mathrm{Mo}^{+}$

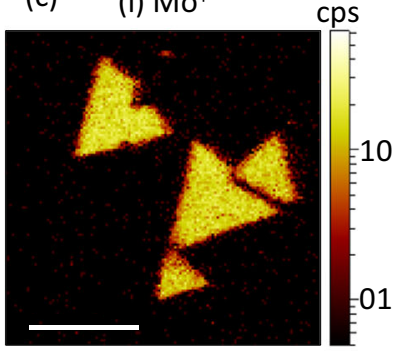

(d)

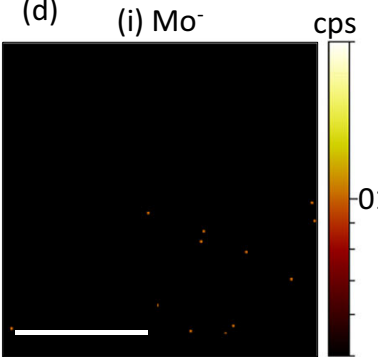

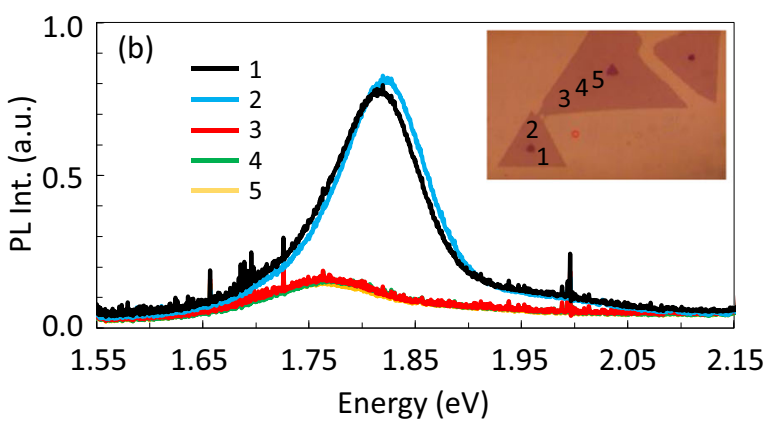

(ii) $\mathrm{S}^{+}$

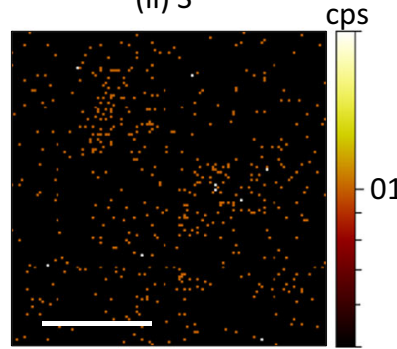

(ii) $\mathrm{S}$

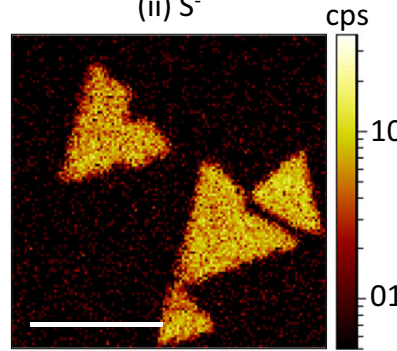

(iii) $\mathrm{Se}^{+}$

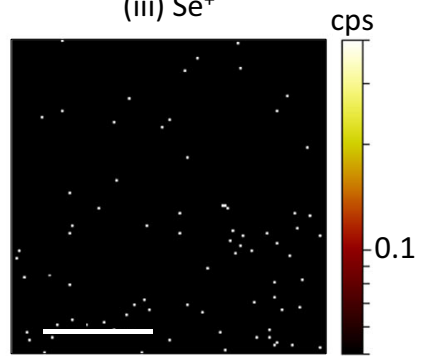

(iii) $\mathrm{Se}^{-}$

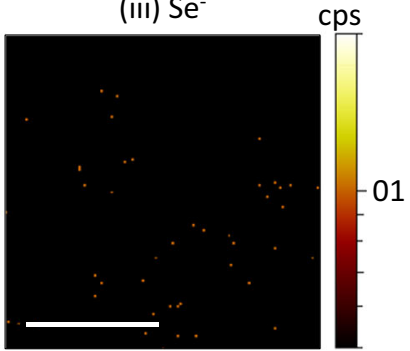

Fig. 5 Elemental analysis of converted $\mathrm{MoS}_{2}$ crystals using ToF-SIMS. a An SEM image of converted MoS $\mathrm{Crystals}$ in which cracked (the bottom) and continuous (three top) triangular films coexist. The inset illustrates a highly magnified image of the region marked by the square. b PL spectra obtained from several points of the $\mathrm{MoS}_{2}$ crystals as marked on the optical image in the inset. The PL spectra of the continuous regions (points 3,4 , and 5) sit on the red side of the spectrum and demonstrate low emission intensities. $\mathbf{c}$, $\mathbf{d}$ Elemental mapping of the converted $\mathrm{MoS}_{2}$ using ToF-SIMS operated in positive and negative modes, respectively. In both modes, investigated ions are (i) molybdenum (Mo), (ii) sulfur (S), and (iii) selenium (Se). The absence of the Se ions in positive and negative scans confirms that the pristine MoSe $e_{2}$ crystals are fully converted to $\mathrm{MoS}_{2}$ in both cracked and continuous regions. Scale bars in all panels are $20 \mu \mathrm{m}$

spectra obtained from the cracked regions to those obtained from CVD-grown samples implies a complete or near-complete stoichiometric composition of the converted $\mathrm{MoS}_{2}$ monolayer crystals. It is worth noting that X-ray photoelectron spectroscopy (XPS) has not been used here for two major reasons. First, visualization in XPS (and generally X-ray systems) is not sufficiently advanced to differentiate between cracked and continuous regions in a randomly distributed array of converted $\mathrm{MoS}_{2}$ crystals. We note that optical imaging (used in XPS) does not distinguish the cracked regions from the continuous ones (e.g., see Fig. 4b). Second, the X-ray beam cannot be well focused on a small spot; thus, XPS provides a relatively low lateral resolution.

In conclusion, we used a two-step composition modulation approach for the synthesis of ternary alloys $\left(\operatorname{MoS}_{2 x} \mathrm{Se}_{2(1-x)}\right)$ and lateral heterostructures $\left(\mathrm{MoSe}_{2}-\mathrm{MoS}_{2}\right)$ within the monolayer crystals of TMDs. Structural analysis revealed that the two-step approach inflicts formation of cracks in the compositionally modulated monolayer crystals, while details of shape and propagation path of the cracks depend on the composition ratio (i.e., $x$ ). Following a set of systematic investigations centered on $\mathrm{PL}$ spectroscopy, we identified the origin of the cracks to be a biaxial tensile strain that stems from the substitution of "Se" atoms by " $\mathrm{S}$ " atoms. Our calculations from the Griffith's criterion demonstrated the substantial contribution of pre-existing cracks in weakening the strength of the synthesized Van der Walls alloys. We believe that our findings significantly contribute to the rapidly growing field of 2D materials as alloying has an irreplaceable role in the fabrication of low-dimensional optoelectronics devices with tunable functionalities. In addition, despite generated as an artifact in the alloying process, cracking renders the assynthesized $\mathrm{MoS}_{2}$ films prime candidates for applications such as hydrogen evolution reactions, ${ }^{40}$ in which abundant edge sites (provided by cracks) significantly enhances the catalytic activity of $\mathrm{MoS}_{2}{ }^{41}$

\section{METHODS}

CVD growth of $\mathrm{MoSe}_{2}$ and synthesis of $\mathrm{MoS}_{2 x} \mathrm{Se}_{2(1-x)}$ alloys Details of monolayer $\mathrm{MoSe}_{2}$ growth process is explained in ref. ${ }^{20}$. Details of the alloying process is presented in the supporting information.

\section{Fabrication of lateral heterostructures}

First, monolayer $\mathrm{MoSe}_{2}$ films were identified via AFM measurements and were used for the formation of lateral heterostructures. Then, standard electron-beam lithography, e-beam evaporation of $70 \mathrm{~nm} \mathrm{SiO} 2$ protecting layer, and lift-off in acetone were sequentially performed to place the sulfurization-protecting masks on the top of the monolayer films. Finally, heterostructures were formed following a sulfurization step. 
Optical characterizations

Raman and PL spectra were collected in a confocal Raman spectrometer (Thermo Nicolet Almega XR), where a $488 \mathrm{~nm}$ laser line was used to excite the sample. In all measurements, the laser was focused to a spot size of approximately $0.6 \mu \mathrm{m}$. The PL mapping was performed with a 1- $\mu \mathrm{m}$ spatial resolution in " $x$ " and " $y$ " directions. To avoid laser-induced heating effects, ${ }^{42}$ the power of the laser was kept below $100 \mu \mathrm{W}$.

\section{ToF-SIMS}

The converted $\mathrm{MoS}_{2}$ samples were characterized using a Bismuth (Bi) primary ion beam at $25 \mathrm{kV}$ acceleration voltage. Specific scans were done using Bi source in burst-bunch mode with $n=10$ at $1 \mu \mathrm{A}$ primary beam current in both positive and negative polarities. To compensate the charging effect, the flood gun was kept constantly ON during the measurements. The mapping resolution was $128 \times 128$ pixels per frame with binning of 1 or 4 depending on different scans.

\section{Data availability}

The datasets generated and/or analyzed during the current study are available from the corresponding author on a reasonable request.

\section{ACKNOWLEDGEMENTS}

This work was primarily funded by the Air Force Office of Scientific Research (AFOSR) under Grant No. FA9550-13-1- 0032 (G. Pomrenke). This work was also supported in part by the Department of Defense, Defense Threat Reduction Agency under contract HDTRA1-16-1-0032, and a Georgia Tech Research Institute (GTRI) Shackelford Fellowship, awarded to P.M. Campbell.

\section{AUTHOR CONTRIBUTIONS}

H.T., A.E., M.T., and A.A. planned the project. H.T. designed the experiments, performed optical characterizations, and conducted structural analysis. H.T., P.M.C., B. B., and C.J.P. contributed in the sulfurization processes. X.Z. and P.A. provided the monolayer $\mathrm{MoSe}_{2}$ films. H.T., M.T., and H.M. fabricated the heterostructures. W.E.H., H. T., and E.V.W. performed the ToF-SIMS experiments. Y.Z. and E.J.R. conducted the Griffith's criterion and D.F.T. calculations. H.T. analyzed the collective results with inputs from A.E. and M.T. H.T. wrote the manuscript with inputs from A.E., M.T., A.A. All authors read the manuscript and provided feedback. A.A., A.E., and E.M.V. supervised the project.

\section{ADDITIONAL INFORMATION}

Supplementary information accompanies the paper on the npj 2D Materials and Applications website (https://doi.org/10.1038/s41699-018-0056-4).

Competing interests: The authors declare no competing interests.

Publisher's note: Springer Nature remains neutral with regard to jurisdictional claims in published maps and institutional affiliations.

\section{REFERENCES}

1. Lopez-Sanchez, O., Lembke, D., Kayci, M., Radenovic, A. \& Kis, A. Ultrasensitive photodetectors based on monolayer $\mathrm{MoS}_{2}$. Nat. Nanotechnol. 8, 497-501 (2013).

2. Wang, X. D. et al. Ultrasensitive and broadband $\mathrm{MoS}_{2}$ photodetector driven by ferroelectrics. Adv. Mater. 27, 6575-6581 (2015).

3. Sundaram, R. S. et al. Electroluminescence in single layer $\mathrm{MoS}_{2}$. Nano Lett. 13, 1416-1421 (2013).

4. Baugher, B. W. H., Churchill, H. O. H., Yang, Y. F. \& Jarillo-Herrero, P. Optoelectronic devices based on electrically tunable $\mathrm{p}-\mathrm{n}$ diodes in a monolayer dichalcogenide. Nat. Nanotechnol. 9, 262-267 (2014).

5. Wu, S. F. et al. Monolayer semiconductor nanocavity lasers with ultralow thresholds. Nature 520, 69-72 (2015).

6. Zhang, M. et al. Two-dimensional molybdenum tungsten diselenide alloys: photoluminescence, Raman scattering, and electrical transport. ACS Nano 8 , 7130-7137 (2014).

7. Tongay, S. et al. Two-dimensional semiconductor alloys: monolayer $\mathrm{Mo}_{1-\mathrm{x}} \mathrm{W}_{\mathrm{x}} \mathrm{Se}_{2}$. Appl. Phys. Lett. 104, 012101 (2014).

8. Duan, X. D. et al. Synthesis of $\mathrm{WS}_{2 x} \mathrm{Se}_{2-2 x}$ alloy nanosheets with compositiontunable electronic properties. Nano Lett. 16, 264-269 (2016).
9. Gong, Y. J. et al. Band gap engineering and layer-by-layer mapping of seleniumdoped molybdenum disulfide. Nano Lett. 14, 442-449 (2014).

10. Li, H. L. et al. Growth of alloy $\operatorname{MoS}_{2 x} \mathrm{Se}_{2(1-x)}$ nanosheets with fully tunable chemical compositions and optical properties. J. Am. Chem. Soc. 136, 3756-3759 (2014).

11. Wang, Z. Q. et al. Chemical vapor deposition of monolayer $M_{1-x} W_{x} S_{2}$ crystals with tunable band gaps. Sci. Rep. 6, 21536 (2016).

12. Zheng, S. J. et al. Monolayers of $\mathrm{W}_{x} \mathrm{Mo}_{1-\mathrm{x}} \mathrm{S}_{2}$ alloy heterostructure with in-plane composition variations. Appl. Phys. Lett. 106, 053113 (2015).

13. Gong, Y. J. et al. Vertical and in-plane heterostructures from $\mathrm{WS}_{2} / \mathrm{MoS}_{2}$ monolayers. Nat. Mater. 13, 1135-1142 (2014).

14. Chen, K. et al. Lateral built-in potential of monolayer $\mathrm{MoS}_{2}-\mathrm{WS}_{2}$ in-plane heterostructures by a shortcut growth strategy. Adv. Mater. 27, 6431-6437 (2015).

15. Duan, X. D. et al. Lateral epitaxial growth of two-dimensional layered semiconductor heterojunctions. Nat. Nanotechnol. 9, 1024-1030 (2014).

16. Mahjouri-Samani, M. et al. Patterned arrays of lateral heterojunctions within monolayer two-dimensional semiconductors. Nat. Commun. 6, 7749 (2015).

17. Kobayashi, Y. et al. Modulation of electrical potential and conductivity in an atomic-layer semiconductor heterojunction. Sci. Rep. 6, 31223 (2016).

18. Su, S. H. et al. Controllable synthesis of band-gap-tunable and monolayer transition-metal dichalcogenide alloys. Front. Energy Res 2, 1-8 (2014).

19. Li, H. L. et al. Composition-modulated two-dimensional semiconductor lateral heterostructures via layer-selected atomic substitution. ACS Nano 11, 961-967 (2017).

20. Wang, X. et al. Chemical vapor deposition growth of crystalline monolayer $\mathrm{MoSe}_{2}$. ACS Nano 8, 5125-5131 (2014).

21. Mouri, S., Miyauchi, Y. \& Matsuda, K. Tunable photoluminescence of monolayer $\mathrm{MoS}_{2}$ via chemical doping. Nano Lett. 13, 5944-5948 (2013).

22. Steinhoff, A. et al. Efficient excitonic photoluminescence in direct and indirect band gap mono layer $\mathrm{MoS}_{2}$. Nano Lett. 15, 6841-6847 (2015).

23. Kang, J., Tongay, S., Li, J. B. \& Wu, J. Q. Monolayer semiconducting transition metal dichalcogenide alloys: stability and band bowing. J. Appl. Phys. 113, 143703 (2013).

24. Hao, S., Yang, B. C. \& Gao, Y. L. Quenching induced fracture behaviors of CVDgrown polycrystalline molybdenum disulfide films. RSC Adv. 6, 59816-59822 (2016).

25. Van der Zande, A. M. et al. Grains and grain boundaries in highly crystalline monolayer molybdenum disulphide. Nat. Mater. 12, 554-561 (2013).

26. Ly, T. H., Zhao, J., Cichocka, M. O., Li, L. J. \& Lee, Y. H. Dynamical observations on the crack tip zone and stress corrosion of two-dimensional $\mathrm{MoS}_{2}$. Nat. Commun. 8, 14116 (2017).

27. Wang, X. N., Tabarraei, A. \& Spearot, D. E. Fracture mechanics of monolayer molybdenum disulfide. Nanotechnology 26, 175703 (2015).

28. Conley, H. J. et al. Bandgap engineering of strained monolayer and bilayer $\mathrm{MoS}_{2}$ Nano Lett. 13, 3626-3630 (2013).

29. Hui, Y. Y. et al. Exceptional tunability of band energy in a compressively strained trilayer $\mathrm{MoS}_{2}$ sheet. ACS Nano 7, 7126-7131 (2013).

30. Zhu, C. R. et al. Strain tuning of optical emission energy and polarization in monolayer and bilayer $\mathrm{MoS}_{2}$. Phys. Rev. B 88, 1-5 (2013).

31. Plechinger, $G$. et al. Control of biaxial strain in single-layer molybdenite using local thermal expansion of the substrate. 2D Mater. 2, 1-8 (2015).

32. Lloyd, D. et al. Band gap engineering with ultra-large biaxial strains in suspended monolayer $\mathrm{MoS}_{2}$. Nano. Lett. 16, 5836-5841 (2015).

33. He, J. G., Hummer, K. \& Franchini, C. Stacking effects on the electronic and optical properties of bilayer transition metal dichalcogenides MoS2, MoSe $2, \mathrm{WS}_{2}$, and WSe ${ }_{2}$. Phys. Rev. B 89, 1-11 (2014).

34. Berkelbach, T. C., Hybertsen, M. S. \& Reichman, D. R. Theory of neutral and charged excitons in monolayer transition metal dichalcogenides. Phys. Rev. B. 88, 1-6 (2013).

35. Bertolazzi, S., Brivio, J. \& Kis, A. Stretching and breaking of ultrathin $\operatorname{MoS}_{2}$. ACS Nano 5, 9703-9709 (2011).

36. Yang, Y. C. et al. Brittle fracture of 2D MoSe 2 . Adv. Mater. 29, 1-7 (2017).

37. Yin, H. Q. et al. Griffith criterion for brittle fracture in graphene. Nano Lett. 15 1918-1924 (2015).

38. Nan, H. Y. et al. Strong photoluminescence enhancement of MoS2 through defect engineering and oxygen bonding. ACS Nano 8, 5738-5745 (2014).

39. Kim, I. S. et al. Influence of stoichiometry on the optical and electrical properties of chemical vapor deposition derived MoS $_{2}$. ACS Nano 8, 10551-10558 (2014).

40. Li, Y. G. et al. $M_{2} S_{2}$ nanoparticles grown on graphene: an advanced catalyst for the hydrogen evolution reaction. J. Am. Chem. Soc. 133, 7296-7299 (2011).

41. Jaramillo, T. F. et al. Identification of active edge sites for electrochemical $\mathrm{H} 2$ evolution from MoS2 nanocatalysts. Science 317, 100-102 (2007).

42. Taghinejad, $\mathrm{H}$. et al. Light-induced heating in hybrid cavity-coupled $2 \mathrm{D}$ transitionmetal dichalcogenides. ACS Photonics 3, 700-707 (2016). 
Open Access This article is licensed under a Creative Commons Attribution 4.0 International License, which permits use, sharing, adaptation, distribution and reproduction in any medium or format, as long as you give appropriate credit to the original author(s) and the source, provide a link to the Creative Commons license, and indicate if changes were made. The images or other third party material in this article are included in the article's Creative Commons license, unless indicated otherwise in a credit line to the material. If material is not included in the article's Creative Commons license and your intended use is not permitted by statutory regulation or exceeds the permitted use, you will need to obtain permission directly from the copyright holder. To view a copy of this license, visit http://creativecommons. org/licenses/by/4.0/.

(c) The Author(s) 2018 